\title{
Efectos de cortas de selección en el crecimiento de brinzales de especies tolerantes latifoliadas: análisis estadístico usando modelos lineales mixtos
}

\author{
Effects of selection cuttings on the growth of saplings of shade-tolerant hardwoods: \\ statistical analysis using linear mixed models.
}

\author{
MARIANO J. DURAN ${ }^{1}$, PABLO J. DONOSO H. ${ }^{2}$, MARIA A. QUINTERO M. ${ }^{3}$ \\ ${ }^{1}$ Universidad de Los Andes, Facultad de Ciencias Forestales y Ambientales, Escuela de Ingeniería Forestal, \\ Departamento de Manejo de Bosques, Mérida, Venezuela. E-mail: mjdurann@ureach.com \\ ${ }^{2}$ Universidad Austral de Chile, Instituto de Silvicultura, Casilla 567, Valdivia, Chile. \\ E-mail:pdonoso@uach.cl \\ ${ }^{3}$ Universidad de Los Andes, Facultad de Ciencias Forestales y Ambientales, Grupo de Investigación y \\ Desarrollo de la Vivienda y el Mueble (GIDEVIM), Mérida, Venezuela. \\ E-mail: mariaq@forest.ula.ve
}

\begin{abstract}
SUMMARY
A statistical analysis by repeated measures was applied to determine height growth patterns of Acer Saccharum Marsh and Fagus grandifolia Ehrh (both shade-tolerant species) growing in a northern hardwood stand under the selection cuttings in North America. The influence of two factors or treatments on height growth was studied: period living under the selection system, also called liberation period, and time elapsed after the first cutting. The methodology used to analyze the data included a General Linear Mixed Model, modeling the covariance matrix. The covariance matrix that best fits the data corresponds to the first order autoregressive structure (AR1). It was demonstrated that the release period and the time after the first cutting significantly affected height growth of trees. The analysis showed that for trees with a liberation period greater than $80 \%$ height growth was significantly higher following the first selection cutting. These results support the effectiveness of the selection system on increasing height growth of young trees. Also, these results recommend a cutting cycle of 15 yrs or less in northern hardwoods under similar conditions to those of this stand.
\end{abstract}

Key words: selection system, cutting cycle, repeated measures, general linear mixed model.

\section{RESUMEN}

Un análisis estadístico con medidas repetidas fue aplicado para determinar patrones de crecimiento en altura de Acer saccharum Marsh y Fagus grandifolia Ehrh (ambas tolerantes a la sombra) creciendo en un rodal de especies latifoliadas en Norteamérica bajo el sistema de selección. Se estudió la influencia de dos factores sobre el crecimiento en altura: el período en que los árboles viven bajo el sistema de selección, también llamado período de liberación, y el tiempo transcurrido después de la primera corta. La metodología utilizada en el análisis de los datos incluyó la aplicación de un modelo lineal mixto y el modelado de la estructura de covarianza de los datos. El modelo de covarianza ajustado corresponde al Autorregresivo de Orden 1 ó AR(1). Se demostró que el período de liberación y el tiempo después de la primera corta influyen sobre el crecimiento en altura de los árboles. Los análisis realizados reflejaron que, para los árboles con período de liberación mayor al $80 \%$, el crecimiento en altura fue significativamente mayor después de la primera corta de selección, demostrándose la efectividad del sistema de selección en árboles jóvenes. Asimismo, los resultados sugieren un ciclo de corta de 15 años o menos para rodales bajo el sistema de selección, bajo las condiciones a las que fue sometido este bosque.

Palabras clave: sistema de selección, ciclo de corta, medidas repetidas, modelo lineal general mixto. 
BOSQUE 26(2): 7-15, 2005

Efectos de cortas de selección en el crecimiento de brinzales de especies tolerantes latifoliadas

\section{INTRODUCCION}

En el este de Norteamérica Acer Saccharum Marsh y Fagus grandifolia Ehrh son dos especies muy comunes de los bosques latifoliados. Por ser especies muy tolerantes a la sombra, son en general dominantes en bosques sucesionales de avanzada edad. Por estas razones, también tienden a ser dominantes en bosques multietáneos manejados bajo el sistema de selección (1). Sin embargo, a pesar de la tolerancia de estas especies, se presume que responden positivamente en crecimiento ante una mayor disponibilidad de recursos. En función de probar este supuesto, el presente artículo examina la respuesta de crecimiento en altura de estas dos especies tras ser sometidas a cortas de selección. En este estudio se evalúa el efecto del período de liberación (proporción de la vida de los árboles que han vivido bajo un sistema de selección mediante cortas cíclicas) y el tiempo transcurrido después de aplicar cortas de selección, sobre el crecimiento en altura y sus interacciones. Igualmente, se compara el crecimiento en altura de los árboles antes y después de realizar la primera corta de selección.

Otro objetivo del estudio fue establecer un modelo estadístico para los efectos de los tratamientos y seleccionar una metodología de análisis apropiada, considerando que la estructura de los datos utilizada involucra medidas repetidas. El término medidas repetidas se refiere a experimentos donde se realizan varias mediciones sobre un mismo individuo (2). En el campo forestal, un experimento con medidas repetidas implica observaciones en intervalos fijos de tiempo (e.g., años, estaciones) o espacio (e.g., profundidad del suelo), para parcelas bajo un régimen de tratamiento (3). En esta investigación, para cada árbol de la muestra se mide el crecimiento en altura en diferentes intervalos de tiempo.

Existen diferentes metodologías estadísticas para el análisis de datos con medidas repetidas. Un primer enfoque sugiere que los datos de cada individuo pueden ser tratados como una serie de tiempo. Sin embargo, en la mayoría de los casos el interés está enfocado en la descripción de los efectos de un tratamiento y cómo éstos varían en el tiempo (4). Littel et al. (5) señalan que existen tres metodologías para estudiar los efectos de un factor en experimentos con medidas repetidas: el análisis de varianza univariante, donde los datos son tratados como si provinieran de un diseño de parcelas divididas; la aplicación de análisis univariantes y multivariantes sobre transformaciones lineales de las medidas repetidas; y un tercer método se basa en un modelo mixto con una estructura paramétrica especial sobre la matriz de covarianza. Aplicaciones de análisis univariante y multivariante en el campo forestal pueden ser estudiadas en los trabajos de Moser et al. (6) y Gompertz y Brownie (7). Meredith y Stehman (3) proponen una metodología basada en el análisis de coeficientes estimados de funciones de las medidas repetidas, como una alternativa más fácil de interpretar que el análisis multivariante.

En este trabajo se utiliza una de las metodologías propuestas por Littel et al. (5), específicamente, aquella donde se usa un modelo lineal mixto y se ajusta una estructura de covarianza especial para los datos. Esta metodología considera la posibilidad de que las observaciones estén correlacionadas, situación que se presenta en experimentos con medidas repetidas, debido a que no es posible asignar aleatoriamente el factor tiempo a las unidades experimentales $(3,6,7)$. En el modelo de efectos mixtos cada vector de respuestas de los individuos (árboles) es modelado como una función paramétrica, donde algunos parámetros o efectos son aleatorios con una distribución normal multivariada. Este modelo ha sido exitoso porque puede ser usado con datos no balanceados $\mathrm{y}$ datos faltantes (8).

\section{MATERIAL Y METODOS}

Area de estudio y características del rodal. El presente estudio se llevó a cabo en un rodal de aproximadamente 9 ha ubicado en el bosque estatal de Cuyler Hill de Nueva Cork, Estados Unidos de Norteamérica. El rodal es dominado por $A$. saccharum y $F$. grandifolia, especies latifoliadas tolerantes a la sombra características del noreste de EE.UU, luego de haber sido sometido a una primera corta de selección en 1973 y una segunda en 1993. El área basal residual fue de $17 \mathrm{~m}^{2} / \mathrm{ha}$ en la primera corta y de $19 \mathrm{~m}^{2} / \mathrm{ha}$ en la segunda. El rodal no fue sometido a intervención alguna durante 20 años antes de la intervención de 1973, salvo por algunas cortas no comerciales de mejoramiento en ciertas partes del rodal. El rodal fue marcado con la finalidad de remover árboles ma- 
duros financieramente, incluyendo la mayoría de aquellos con diámetro a la altura de pecho (DAP) superior a $56 \mathrm{~cm}$, y de balancear la distribución diamétrica de acuerdo con la estructura residual recomendada por Arbogast (9). La marcación favoreció a los árboles de más vigor y mejor calidad fustal. El rodal residual en promedio tuvo $3.5 \mathrm{~m}^{2} / \mathrm{ha}$ en área basal menos que aquella recomendada por Arbogast (9), debido principalmente a insuficiencia de árboles mayores a $56 \mathrm{~cm}$ de DAP y a deficiencias en árboles pequeños ( $<23 \mathrm{~cm}$ de DAP).

Recolección de la muestra y análisis del crecimiento en altura. Con anterioridad el rodal había sido dividido en 54 cuadrados de 31 x $31 \mathrm{~m}$. En el año 1997, fueron cortados los árboles muestra para este estudio. Se seleccionaron aleatoriamente 20 cuadrados de un total de 54 al interior del rodal, y en cada uno de ellos se cortó un brinzal (árboles entre 2.5 y $5 \mathrm{~cm}$ de DAP) de $A$. saccharum y uno de $F$. grandifolia. El criterio de selección fue considerar los árboles de cada especie más cercanos al centro del cuadrado. Siguiendo este procedimiento se obtuvo una muestra de 20 árboles de A. saccharum y 20 de $F$. grandifolia.

Antes de cortar los árboles, el DAP fue marcado y medido. Luego, a cada árbol se le midió la altura total. Una vez cortados los árboles, éstos fueron seccionados en segmentos de $30 \mathrm{~cm}$ de largo, comenzando desde la altura del suelo o tan cerca de ésta como fuera posible. Se obtuvieron segmentos hasta la última sección posible, lo cual en general correspondió a unos 30 ó $60 \mathrm{~cm}$ bajo la punta. En el laboratorio luego se midió la edad de cada sección. La sección correspondiente al DAP también fue medida. El origen de los árboles no fue determinado, debido a la dificultad de hacer esta determinación en árboles de A. saccharum de más de $1 \mathrm{~cm}$. de DAP (11) y en árboles de $F$. grandifolia con DAP superior a $2.5 \mathrm{~cm}$. Debido a que la primera corta de selección se aplicó al rodal con 24 años de anterioridad a 1997, los árboles se dividieron en tres categorías, de acuerdo a la proporción de tiempo que crecieron bajo condiciones de liberación o manejo. Las categorías consideradas incluyeron aquellos árboles con más de un $80 \%$ de sus vidas creciendo bajo condiciones de manejo (árboles jóvenes), los cuales correspondieron a árboles de 26 a 30 años de edad (categoría 1); árbo- les creciendo entre un $67 \%$ y $80 \%$ de su vida bajo condiciones de manejo (árboles de edad intermedia), que correspondieron a árboles de 31 a 36 años de edad (categoría 2); y árboles que han vivido menos del $67 \%$ de sus vidas bajo condiciones de manejo (árboles viejos), o de 37 o más años de edad (categoría 3). El tamaño muestral para los árboles de categoría 1 fue de 12, para la categoría 2 de 11, y para la categoría 3 de 16.

Para cada árbol se determinó el crecimiento periódico en altura para el primer ciclo de corta. Los períodos analizados correspondieron a: período 1 (1973-1978), período 2 (1978-1983), período 3 (1983-1988), período 4 (1988-1993). En realidad el ciclo de corta se extendió hasta 1992, pero debido a que se usó el crecimiento en altura para períodos de 5 años, la información para el período 1988-1993 fue calculada a partir del crecimiento en el período 1988-1992 multiplicado por 5/4, estimándose así el crecimiento en 5 años. Adicionalmente, el período (1968-1973) fue considerado como control o condición de no manejo. Para este período fue posible obtener información de todos los árboles de al menos 29 años de edad. Para los árboles con edades comprendidas entre 26 y 28 años, la información fue extrapolada a partir de los datos disponibles.

Modelo estadístico utilizado. En este estudio la variable respuesta está dada por el crecimiento en altura de dos especies de árboles ( $A$. saccharum y $F$. grandifolia) que fueron sometidas a un sistema de manejo de cortas de selección. Hay dos factores que influyen en el crecimiento en altura: un factor entre unidades que es la proporción de tiempo que el árbol estuvo bajo condiciones de manejo, llamado período de liberación, y un factor dentro de unidades, llamado tiempo. El factor período de liberación tiene tres tratamientos (categorías), y cada árbol recibe uno solo de ellos. El factor tiempo representa medidas repetidas en cada árbol, ya que para un árbol hay cinco medidas de altura, que corresponden al crecimiento en diferentes intervalos de tiempo. Estudios previos indicaron que no existe diferencia significativa entre los patrones de crecimiento de las dos especies, por lo tanto, no se consideró la especie como un factor que influye en el crecimiento en altura (11).

El análisis utilizado corresponde a un modelo bajo el esquema de parcelas divididas con 
medidas repetidas, donde las unidades experimentales son los árboles, las tres categorías del período de liberación son los tratamientos de las parcelas (tratamiento principal), los cinco períodos de tiempo son los tratamientos de las subparcelas, y la variable respuesta es el crecimiento en altura. El modelo estadístico es el siguiente:

$$
Y_{i j k}=\mu+a_{i}+d_{i j}+b_{j}+(a b)_{i j}+e_{i j k} \mathrm{i}=1,2,3 ; \mathrm{j}=1,2, \ldots, 5 ; \mathrm{k}=1,2, \ldots, 39
$$

Donde:

$\mathrm{Y}_{\mathrm{i} \text { j } \mathrm{k}}$ : crecimiento en altura del k-ésimo árbol del período de liberación i en el tiempo j.

$\mu \quad$ : media general

$\mathrm{a}_{\mathrm{i}} \quad$ : efecto de la categoría i del período de liberación

$\mathrm{d}_{\mathrm{ij}} \quad$ : efecto aleatorio asociado al j-ésimo árbol en la categoría $\mathrm{i}$

$b_{j} \quad$ : efecto del período de tiempo $j$

$(\mathrm{ab})_{\mathrm{ij}}$ : efecto de la interacción entre el período de liberación i y el tiempo j.

$\mathrm{e}_{\mathrm{ijk}} \quad$ : error aleatorio asociado al k-ésimo árbol del período de liberación i en el tiempo j.

En un diseño de parcelas divididas clásico, que no considera medidas repetidas, se asumen errores independientes, lo que significa que todas las observaciones están, al menos, igualmente correlacionadas. Sin embargo, cuando se trabaja con medidas repetidas, como en este caso, las observaciones de puntos cercanos en el tiempo usualmente están más correlacionadas entre sí que las observaciones que están más distanciadas en el tiempo (12). Para verificar esta aseveración, se calculó el coeficiente de correlación de Pearson entre las observaciones en los diferentes intervalos de tiempo.

Esta situación hace necesario modelar la estructura de covarianza de los datos, de modo que las inferencias acerca de las medias sean las apropiadas. Para ajustar el modelo estadístico planteado y modelar la estructura de covarianza, se utilizó el PROC MIXED del paquete estadístico SAS (SAS Institute Inc., 1999-2000). El PROC MIXED ofrece varias estructuras de covarianzas, de las cuales las más comúnmente usadas son: simétrica compuesta (compound symmetric, CS), la cual asume que la correlación entre dos observaciones es la misma sin importar su distanciamiento en el tiempo; autorregresiva de orden 1 ó AR(1), donde las observaciones correspondientes a puntos del tiempo cercanos tienen una correlación mayor que aquellas observaciones que están distanciadas en el tiempo; y covarianza sin estructura (unstructured, UN), donde no se impone un patrón matemático a la matriz de covarianza (5). El programa SAS que permite modelar la estructura de la matriz de covarianza se muestra a continuación:
Proc mixed data $=$ crecim class plib árbol tiempo model altura $=$ plib tiempo plib*tiempo repeated $/$ type $=\operatorname{ar}(1)$ sub=arbol(plib) lsmeans tiempo*plib run

En la declaración REPEATED, Type $=\operatorname{ar}(1)$ especifica la estructura de covarianza de tipo autorregresiva de orden 1. La opción sub=árbol (plib) especifica que la estructura de covarianza $\operatorname{ar}(1)$ corresponde a cada árbol en cada tratamiento (categoría del período de liberación). El comando sub=árbol(plib) indica que la estructura de la matriz simétrica compuesta pertenece a las submatrices correspondientes a cada árbol dentro de cada período de liberación.

Para elegir la estructura de covarianza que mejor se ajustara a los datos, se tomaron en consideración dos criterios que calcula el PROC MIXED: Criterio de Información de Akaike (AIC) y Criterio de Información Bayesiano de Schwartz (BIC). La estructura de covarianza con valores de los criterios más cercanos a cero se considera más apropiada para obtener inferencias válidas de los efectos fijos (5).

Una vez establecida la estructura de covarianza más adecuada, se procedió a analizar los resultados del análisis de varianza, los cuales permitieron evaluar si los efectos de los factores período de liberación, tiempo y su interacción sobre el crecimiento en altura eran estadísticamente significativos. Adicionalmente, se realizaron las pruebas de normalidad de Shapiro-Wilk, Kolmogo- 
rov-Smirnov, Cramer-Von Mises y Anderson-Draling, para determinar si los residuos se distribuyen normalmente. De esta forma se verificó la idoneidad del modelo estadístico empleado.

\section{RESULTADOS Y DISCUSION}

Modelado de la estructura de covarianza y pruebas de idoneidad del modelo. Antes de modelar la estructura de covarianza de los datos, se calculó el coeficiente de correlación de Pearson entre las observaciones, así como las varianzas en el tiempo, usando el PROC CORR de SAS. Las correlaciones entre las observaciones cambian en el tiempo (cuadro 1). Para los tiempos 2, 3, 4 y 5, las observaciones adyacentes tienen mayor correlación que aquellas que están más alejadas. En cuanto a la varianza, ésta, en general, tiende a disminuir conforme aumenta el tiempo de medición.

Una vez analizadas las correlaciones en el tiempo, se probaron las tres estructuras de covarianza antes señaladas, haciendo uso del PROC MIXED. Los criterios AIC y BIC obtenidos para cada modelo de estructura de covarianza se presentan en el cuadro 2. De acuerdo a estos criterios, se selecciona como estructura de covarianza más adecuada para los datos originales la autorregresiva de orden 1 ó $\operatorname{AR}(1)$.

Pruebas de normalidad de los residuos del modelo, usando la estructura de covarianza AR(1), indicaron que los residuos no siguen una distribución normal, tal como se observa en el cuadro 3.
Por esta razón, fue necesario aplicar una transformación logarítmica a los datos originales.

Transformación de los datos. Con el logaritmo del crecimiento en altura como variable respuesta, se procedió a analizar las correlaciones y las varianzas de las observaciones en el tiempo. Se evidencia, al igual como ocurrió con los datos originales, que las observaciones no están igualmente correlacionadas (cuadro 1). Se presenta una mayor correlación entre puntos adyacentes en el tiempo, que entre aquellos que están más distanciados. Asimismo, puede notarse que las varianzas en el tiempo (elementos de la diagonal de la matriz) tienden a estabilizarse, lo que se debe a la transformación logarítmica aplicada a los datos.

Posteriormente, se seleccionó la estructura de covarianza más adecuada, de la misma forma que se hizo con los datos originales. Los valores de los criterios de decisión se muestran en el cuadro 2. De acuerdo a estos criterios, se selecciona como estructura de covarianza para los datos transformados la autorregresiva de orden 1 AR(1). La estructura de covarianza más adecuada es la misma para el modelo con el crecimiento en altura y para el modelo con el logaritmo del crecimiento en altura.

Al efectuar pruebas de normalidad a los residuos, se pudo comprobar que dicho supuesto se cumple usando los datos transformados, tal como se observa en el cuadro 3, lo que indica que el modelo estadístico planteado es válido. Cabe destacar que los supuestos de homocedasticidad e

\section{CUADRO 1}

Coeficientes de correlación de Pearson (dos colas) para los datos de crecimiento en altura durante distintos períodos de tiempo, para los datos originales (letra normal) y transformados (letra cursiva); en paréntesis valores de $\mathrm{p}$.

Two-tailed Pearson's correlation coefficient for the original (in normal letter) and transformed (in italics) data in height growth in different time periods.

\begin{tabular}{|cccccc|}
\hline & Tiempo 1 & Tiempo 2 & Tiempo 3 & Tiempo 4 & Tiempo 5 \\
\hline Tiempo 1 & 1 & $0,062(0,709)$ & $-0,151(0,360)$ & $-0,191(0,243)$ & $-0,292(0,071)$ \\
Tiempo 2 & $0,080(0,630)$ & 1 & $0,200(0,223)$ & $0.090(0,587)$ & $0,086(0,604)$ \\
Tiempo 3 & $0,053(0,748)$ & $0,106(0,522)$ & 1 & $0,504(0,001)$ & $-0,090(0,587)$ \\
Tiempo 4 & $-0,163(0,322)$ & $-0,022(0,893)$ & $0,451(0,004)$ & 1 & $0,311(0,054)$ \\
Tiempo 5 & $-0,266(0,102)$ & $0,073(0,659$ & $-0,139(0,399)$ & $0,335(0,037)$ & 1 \\
\hline
\end{tabular}


BOSQUE 26(2): 7-15, 2005

Efectos de cortas de selección en el crecimiento de brinzales de especies tolerantes latifoliadas

independencia no fueron evaluados, debido a que se utilizó una estructura de covarianza del tipo $\mathrm{AR}(1)$.

\section{CUADRO 2}

Valores de los criterios de decisión para las estructuras de covarianza modeladas usando los datos originales y transformados.

Values for the decision criteria used for the modeled covariance structures, using the original and the transformed data.

\begin{tabular}{|lllll|}
\hline Tipo de datos & $\begin{array}{l}\text { Criterio de } \\
\text { información }\end{array}$ & $\mathrm{CS}^{1}$ & $\mathrm{AR}(1)^{2}$ & $\mathrm{UN}^{3}$ \\
\hline Originales & Akaike (AIC) & 564,6 & 562,0 & 559,5 \\
& Bayesiano (BIC) & 571,9 & 565,3 & 584,4 \\
Transformados & Akaike (AIC) & 244,1 & 235,4 & 243,6 \\
& Bayesiano (BIC) & 247,4 & 238,7 & 268,5 \\
\hline
\end{tabular}

${ }^{1}$ CS: Simétrica Compuesta.

${ }^{2}$ AR(1): Autorregresiva de orden 1.

${ }^{3} \mathrm{UN}$ : Covarianza sin estructura.

\section{CUADRO 3}

Resultados de las pruebas de normalidad de los residuos, usando los datos originales y transformados (valores $\mathrm{p}$ ).

Results of the normality test of the residuals, using original data and transformed data ( $\mathrm{p}$-values).

\begin{tabular}{|lcc|}
\hline Prueba & $\begin{array}{c}\text { Datos } \\
\text { Originales }\end{array}$ & $\begin{array}{c}\text { Datos } \\
\text { Transformados }\end{array}$ \\
\hline Shapiro-Wilk & 0,0001 & 0,2067 \\
Kolmogorov-Smirnov & 0,0100 & 0,0600 \\
Cramer-Von Mises & 0,0050 & 0,0901 \\
Anderson-Draling & 0,0050 & 0,1162 \\
\hline
\end{tabular}

Nivel de significancia: $5 \%$.

Análisis de varianza. De los valores obtenidos del análisis de varianza (cuadro 4) se desprende que el efecto de la interacción es significativo a un nivel de significancia del 5\%; esto es, la interacción entre el período de liberación y el tiempo influye en el crecimiento en altura. El efecto del período de liberación también es significativo a un nivel de significancia del 5\%, es decir, que el período en que los árboles han vivido bajo el sistema de selección influye en el crecimiento en altura. El efecto del tiempo igualmente resultó significativo al mismo nivel, lo que indica que el tiempo transcurrido antes y después de la primera corta de selección influye en el crecimiento en altura de árboles que viven bajo este sistema de manejo.

\section{CUADRO 4}

Resultados del Análisis de Varianza,

con los datos transformados (log) y una estructura de covarianza AR(1).

Results of the Analysis of Variance with the log-transformed data and a AR (1) covariance structure.

\begin{tabular}{|lccc|}
\hline Fuente de Variación & $\begin{array}{c}\text { Grados } \\
\text { de libertad }\end{array}$ & F & Valor p \\
\hline $\begin{array}{l}\text { Entre tratamientos } \\
\text { Período de liberación }\end{array}$ & 2 & 5,10 & 0,0112 \\
$\begin{array}{l}\text { Error A } \\
\text { Dentro de tratamientos }\end{array}$ & 36 & & \\
$\begin{array}{l}\text { Tiempo } \\
\text { Tiempo x período } \\
\text { de liberación }\end{array}$ & 4 & 2,99 & 0,0209 \\
Error B & 8 & 2,09 & 0,0402 \\
\hline
\end{tabular}

Complementariamente, se construyó un gráfico del logaritmo del crecimiento en altura promedio para cada combinación de período de liberación y tiempo (figura 1), en el cual se observa que existen diferencias en el crecimiento en altura de los árboles para los distintos intervalos de tiempo y períodos de liberación. Existe un aumento en la tasa de crecimiento de los árboles jóvenes (período de liberación $>80 \%$ ) cuando comienza el sistema de selección, tiempo 2 (1973-1978); lo mismo ocurre con los árboles de edad intermedia (período de liberación entre $67,7 \%$ y $80 \%$ ).

En el tiempo 1 (período sin manejo), los árboles jóvenes tienen un crecimiento menor que los árboles viejos y el mayor crecimiento se registra en los árboles de edad intermedia. A partir del tiempo 2, momento en que se realiza la primera corta de selección, los árboles que han vivido más de $67,7 \%$ de sus vidas bajo manejo (árboles jóvenes e intermedios) presentan valores mayores de crecimiento en altura que los árboles viejos. 
BOSQUE 26(2): 7-15, 2005 Efectos de cortas de selección en el crecimiento de brinzales de especies tolerantes latifoliadas

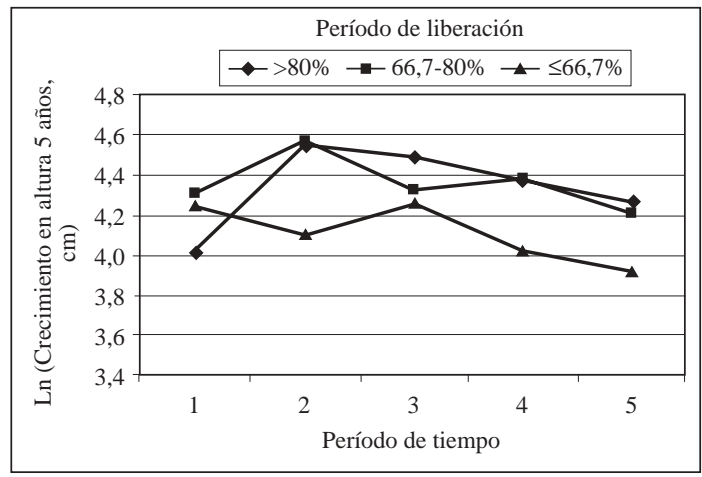

Figura 1. Logaritmo del crecimiento en altura promedio para cada combinación de tiempo y período de liberación.

Logarithm of height growth for each combination of time and release period.

Análisis de contrastes. En vista de que el efecto del tiempo, el efecto del período de liberación y la interacción son significativos, se realizó un análisis a posteriori para determinar cuáles son las medias que difieren entre sí. Se probaron contrastes con la opción "contrast" del SAS. Mediante este comando se realizan pruebas F para determinar diferencias entre tratamientos, con el fin de establecer si existe diferencia entre la media en el tiempo 1 (control) versus el promedio de las medias en los tiempos 2, 3, 4 y 5, dejando fijo el período de liberación (cuadro 5). Al analizar los contrastes, se observó que, si se fija el período de liberación en 1, existen diferencias significativas entre la media registrada en el tiempo 1 (19681973), antes de realizarse la primera corta de selección, y el promedio de las medias correspondientes a los tiempos 2, 3, 4 y 5. Esto significa que el sistema aplicado es efectivo en árboles que han vivido más del $80 \%$ de sus vidas bajo el sistema de selección. Lo contrario ocurre cuando se mantiene fijo el período de liberación en 2 y 3 . En estos casos no existe diferencia significativa entre el tiempo control y los tiempos en los cuales se aplicó el sistema de selección; esto indica que el sistema de selección no fue efectivo en árboles que han vivido menos del $80 \%$ de sus vidas bajo manejo.

De igual manera, se hicieron comparaciones por pares de medias para el factor tiempo, dejando fijo el período de liberación (cuadro 5). Puede notarse que para el período de liberación 1 (árbo-

\section{CUADRO 5}

Contrastes para el efecto tiempo, dejando fijo el período de liberación.

Contrasts for time effect and dates for the release period.

\begin{tabular}{|lccc|}
\hline & \multicolumn{3}{c|}{ Período de liberación (PL) } \\
\cline { 2 - 4 } $\begin{array}{l}\text { Contrastes } \\
\text { para el tiempo }\end{array}$ & $\begin{array}{c}2 \\
\text { PL>80\% }\end{array}$ & $\begin{array}{c}66,7 \% \\
\text { <PL }<80 \%\end{array}$ & PL $<66,7 \%$ \\
\hline 1 vs. Promedio $(2,3,4,5)$ & $0,0031^{*}$ & 0,6549 & 0,1364 \\
1 vs. 2 & $0,0005^{*}$ & 0,1030 & 0,2784 \\
1 vs. 3 & $0,0056^{*}$ & 0,9028 & 0,9414 \\
1 vs. 4 & $0,0395^{*}$ & 0,6837 & 0,1202 \\
1 vs. 5 & 0,1597 & 0,5719 & $0,0285^{*}$ \\
2 vs. 3 & 0,6700 & 0,1346 & 0,2439 \\
2 vs. 4 & 0,2882 & 0,2936 & 0,5334 \\
2 vs. 5 & 0,0977 & $0,0465 *$ & 0,2057 \\
3 vs. 4 & 0,4476 & 0,7423 & 0,0641 \\
3 vs. 5 & 0,1879 & 0,4818 & $0,0201^{*}$ \\
4 vs. 5 & 0,4766 & 0,2664 & 0,4517 \\
\hline
\end{tabular}

*: El contraste es significativo a un nivel del $5 \%$.

les jóvenes), hay diferencias en el crecimiento en altura de los árboles entre el tiempo $1 \mathrm{y}$ los tiempos 2, 3 y 4. Entre el tiempo 1 (período de no manejo) y el tiempo 5 (antes de realizar la segunda corta de selección), no hay diferencia significativa entre las medias del crecimiento en altura; esto indica que el efecto positivo del sistema de selección sobre el crecimiento en altura de los árboles se pierde durante los últimos 5 años dentro del ciclo de corta de 19 años.

Para el período de liberación 2 (árboles de edad intermedia), sólo hay diferencias entre las medias de los tiempos 2 y 5 . De esto puede deducirse que el crecimiento en altura, durante el primer período de cinco años después de la primera corta, es considerablemente mayor que el crecimiento en altura durante el último período de la corta de selección.

Para el período de liberación 3, hay diferencias significativas entre las medias de los tiempos 1 y 5 , y entre las medias de los tiempos 3 y 5 ; el crecimiento en altura fue mayor en el período de no manejo (tiempo 1) y en el tiempo 3 , que en el tiempo 5 . 
BOSQUE 26(2): 7-15, 2005

Efectos de cortas de selección en el crecimiento de brinzales de especies tolerantes latifoliadas

\section{CONCLUSIONES}

El análisis de los datos permitió obtener las siguientes conclusiones, acerca del comportamiento del crecimiento en altura de árboles de $A$. saccharum y $F$. grandifolia, tras ser sometidos a un sistema de selección:

Se determinó que el tiempo transcurrido después de la primera corta de selección afecta en forma significativa el crecimiento en altura de los árboles. En general, a medida que transcurre el tiempo va disminuyendo el crecimiento en altura. Se observa mayor crecimiento en altura durante los primeros 15 años después de la primera corta que para los restantes 4 años antes de la segunda corta. Esto sugiere usar un máximo de 15 años como ciclo de corta para rodales bajo el sistema de selección y bajo las condiciones a las que fue sometido este bosque, incluyendo la dominancia de A. saccharum y $F$. grandifolia, dos especies muy tolerantes a la sombra.

La proporción de sus vidas que los árboles están bajo manejo, o período de liberación, es una variable que también influye sobre el crecimiento en altura. Los árboles que han vivido más del $80 \%$ de sus vidas con el sistema de selección muestran un mayor crecimiento en altura que árboles con períodos de liberación menor al $80 \%$. El menor crecimiento en altura se presenta en árboles con período de liberación menor al $67,7 \%$

La interacción entre las dos variables en estudio, tiempo y período de liberación, afecta el crecimiento en altura. Como consecuencia de esta interacción, se demostró que para los árboles con un período de liberación mayor al $80 \%$, que son árboles que tienen de 26 a 30 años de edad, existe diferencia significativa entre la media registrada en el tiempo 1 (1968-1973) o condición de no manejo y el promedio de las medias correspondientes a los tiempos 2, 3, 4 y 5, después de realizar la primera corta de selección. Este resultado refleja la conveniencia y efectividad del sistema de selección en árboles jóvenes de las dos especies en estudio.

Para árboles cuyo período de liberación fue menor al $80 \%$, se determinó que no existen diferencias estadísticamente significativas entre el crecimiento en altura antes y después de aplicar la primera corta de selección. Por lo tanto, sobre la base de los datos de este estudio, no se puede establecer que al menos una primera corta de selección sea efectiva en árboles con edades superiores a los 30 años.

Las tendencias obtenidas reflejan la capacidad de especies tolerantes a la sombra de responder al buen manejo forestal, en este caso, a través de cortas de selección. Estos resultados son alentadores no sólo para estos bosques de latifoliadas de zonas templadas de Norteamérica, sino que para otros bosques templados en que las especies tolerantes son importantes en abundancia y valor comercial. En el caso de Chile, por ejemplo, tepa (Laureliopsis philippiana) y olivillo (Aextoxicon punctatum) son dos especies tolerantes (13), de gran uso (14), y particularmente tepa de una extensa distribución geográfica (13).

En cuanto al método estadístico utilizado, se concluye que en el análisis de experimentos con medidas repetidas es necesario emplear modelos lineales mixtos, debido a que las correlaciones entre las mediciones hechas sobre un mismo individuo no son iguales. El modelado de la estructura de covarianza es un paso preliminar en el análisis de medidas repetidas usando modelos mixtos y es esencial para obtener inferencias válidas sobre las medias; si no se considera la forma como se correlacionan las observaciones en experimentos de este tipo, podría llegarse a conclusiones erróneas. Es por ello, que la metodología aplicada en este estudio se recomienda para analizar los resultados de experimentos en los cuales se toman varias mediciones en el tiempo sobre las mismas unidades experimentales.

\section{BIBLIOGRAFIA}

(1) NYLAND, R.D., P.J. CRAUL, D.F. BEHREND, H.E. ECHELBERG, W.J. GABRIEL, R.L. NISSEN, R. UEBLER, J. ZARNETSKE. Logging and its effects in northern hardwoods. SUNY College of Environmental Science and Forestry, Applied Forest Research Institute, AFRI Research Report No 31, Syracuse, NY, USA. 1976. 27 p.

(2) HAYS, W. Statistics. Hoet, Rinehart and Winston, Inc. New York, USA. 1988. 1029 p.

(3) MEREDITH, M. P., S.V. STEHMAN. Repeated measures experiments in forestry: focus on analysis of response curves. Canadian Journal of Forest Research, 1991, Vol. 21, p. 957-965.

(4) VERBYLA A. P., B.R. CULLIS. Modelling in repeated measures experiments. Applied Statistics, 1990, Vol. 39, $\mathrm{N}^{\circ} 3$, p. 341-356.

(5) LITTEL R., G. MILLIKEN, W. STROUP, R. WOLFIGER. SAS System for Mixed Models. SAS Institute Inc, NC, USA. 1996. 633 p. 
BOSQUE 26(2): 7-15, 2005 Efectos de cortas de selección en el crecimiento de brinzales de especies tolerantes latifoliadas

(6) MOSER, E. B., A.M. SAXTON, S.R. PEZESHKI. Repeated measures analysis of variance: application to tree research. Canadian Journal of Forest Research, 1990, Vol. 20 (5), p. 524-535.

(7) GOMPERTZ, M.L., C. BROWNIE. Repeated measures in randomized block and split-plot experiments. Canadian Journal of Forest Research, 1993, Vol. 2, p. 625-639.

(8) LINDSTROM, M.J., D. BATES. Newton-Raphson and EM algorithm for linear mixed-effects model for repeated measures data. Journal of the American Statistical Association, 1988, Vol.83 (404), p. 1014-1022.

(9) ARBOGAST, C. Jr. Marking guides for northern hardwoods under the selection system. USDA For. Serv. Lake States For. Exp. Sta., 1957, Station Paper 56, 19 p.
(11)HETT, J.M., O.L. LOUCKS. Sugar maple (Acer saccharum Marsh.) seedling mortality. Ecology, 1971, Vol. 59, p. 507-520.

(11)DONOSO, P. Assessment of regeneration composition growth and ingrowth in the Cuyler Hill Selection System Stand through 24 years and two cuttings. M.Sc.

Thesis, SUNY College of Environmental Science and Forestry, Syracuse, NY, USA. 1998. 98 p.

(12) FINNEY, D.J. Repeated measures: what is measured and what repeats? Stat. Med. 1990, Vol. 9, p. 639-644.

(13) DONOSO, C. Estructura, Variación y Dinámica de Bosques Templados de Chile y Argentina. Ecología Forestal. Ed. Universitaria, Santiago, Chile. 1993. 484 p.

(14) HALL, M., J. WITTE. Maderas del sur de Chile. IER Ediciones, Santiago, Chile. 1998. 91 p.

Recibido: 07.10 .03

Aceptado: 13.09 .04 